\title{
Research on the Action Mechanism of Cultural and Creative Industries on Urban Economic Growth under the Background of Media Convergence
}

\author{
Xinyu Liu \\ School of Performance and Cultural Industries, University of Leeds, LS2 9JT, United Kingdom \\ 453319012@qq.com
}

\begin{abstract}
Cultural and creative industries interact with the urban economy. Their growth can not only change the mode of economic growth, promote the optimization and upgrading of industrial structure, solve employment problems, directly affect the urban economy, but also indirectly drive economic growth through technological innovation, human capital and cultural values. At present, Media convergence has become the general trend, and technological innovation has weakened the boundaries and barriers of the traditional media market. The media market has been expanded not only in geographical concept but also in product categories. "The integration of technology has blurred the division of different kinds of media, communication products and markets". In this paper, the mechanism model of cultural and creative industries on urban economy is established by constructing structural equations, and then the mechanism of cultural and creative industries is more fully understood.
\end{abstract}

Keywords: Media Convergence; Cultural and Creative Industries; Urban Economy.

\section{Introduction}

Media convergence has become a mainstream trend today, and the rise of new media has not caused the decline of traditional media. On the contrary, while the industry barriers between the two have become more and more blurred, business crossover and cooperation have become more frequent, extensive and in-depth. Media convergence, resource sharing, and complementary advantages have enabled the audience's needs to be more fully met through a variety of ways, and various resources have been integrated and used to a greater extent, so that the value chain of the cultural and creative industry has been continuously extended and expanded. Traditional media and new media have opened a win-win space [1]. Cultural and creative industry is an emerging industry in the national economic system. It has the characteristics of innovation, permeability, high added value and strong radiation. It has been widely concerned and highly praised by countries all over the world. In recent years, cultural and creative industries have also developed rapidly and become a strategic choice to change the mode of economic growth, promote the optimization and upgrading of industrial structure, solve employment problems, strengthen national cohesion and improve social values [2]. Cultural and creative industries are closely linked with urban economy, and cities are ideal places for the development of creative industries, which also inject new impetus into urban development [3]. Many scholars at home and abroad have paid attention to the problem that creative industries promote urban economic growth and social construction and development. However, most of them are based on theoretical analysis, with few empirical analyses, and few scholars are involved in cultural and creative industries and urban economic analysis. In view of the deficiency of current research, this paper puts forward the research on the action mechanism of cultural and creative industries on urban economic growth under the background of Media convergence, to form a reference for the development of creative industries. 


\section{Analysis of the Mechanism of Cultural and Creative Industries on Urban Economy under the Background of Media Convergence}

\subsection{Media Convergence}

At present, Media convergence has become the general trend. Technological innovation has weakened the boundaries and barriers of the traditional media market. The media market has expanded not only in terms of geographic concepts, but also in product categories. "The integration of technology enables different types of media, communication products, and the division of the market has become blurred". Examining the domestic market, in the process of Media convergence centered on the "triple play", six major value creations for the upstream and downstream industrial chain have been formed with content-platform-transmission-screen-consumers and equipment providers. Link. In the traditional sense, media content providers have transformed into cross-media service providers, and transnational capital has appeared more in this industry chain link [4].

A perspective to observe the change of competition pattern of large media industry is the change of ownership. In a changing and transitional society, the change of economic ownership is the most decisive factor. Here, we mainly focus on the ownership changes related to the TV new media industry and the relationship between this change and the whole cultural and creative industry.

\subsection{Analysis of the Mechanism of Creative Industries' Effect on Urban Economy}

Cultural and creative industry is the product of the blending of culture, economy and politics. Besides its own contribution to economic growth, it can also establish a coupling relationship with the influencing factors of economic growth, such as technological innovation and human capital, thus indirectly driving economic growth. Creative industry has the characteristics of growth, innovation and high added value, which not only accumulates its wealth rapidly but also drives related links to grow into a complete industrial chain, which directly promotes the economic development of cities, and at the same time opens up a brand-new development mode of mobilizing cultural resources and integrating economic resources [5].

Cultural and creative industries are transformed into new technologies through human creativity input, and then into high value-added products and services, driving huge industrial profits and promoting the development of regional economy. The cultural and creative industries not only directly promote the urban economy, but also indirectly promote the urban economy through innovation mechanisms, diffusion and spillover mechanisms, and transformation mechanisms, which are mainly reflected in the following three aspects: Cultural and creative industries use technology Innovation and knowledge accumulation play a role in economic growth. The cultural and creative industries have effectively improved human capital. The cultural creative industry acts on economic growth through cultural values [6].

\subsection{Cultural and Creative Industries and Urban Economic Development}

Along the industrial development path, cultural and creative industries and urban economic development along the economic development path, continue to evolve and develop in depth and dynamically. In this evolution and development process, factor agglomeration plays an important role in the interaction at different stages [7]. At different stages of evolution and development, the operation mode, operation state and characteristics of the elements gathered in the interactive relationship between cultural and creative industries and urban economic development are also different. The action mechanism of the elements gathered in the interactive evolution and development of the two systems is shown in Figure 1. 


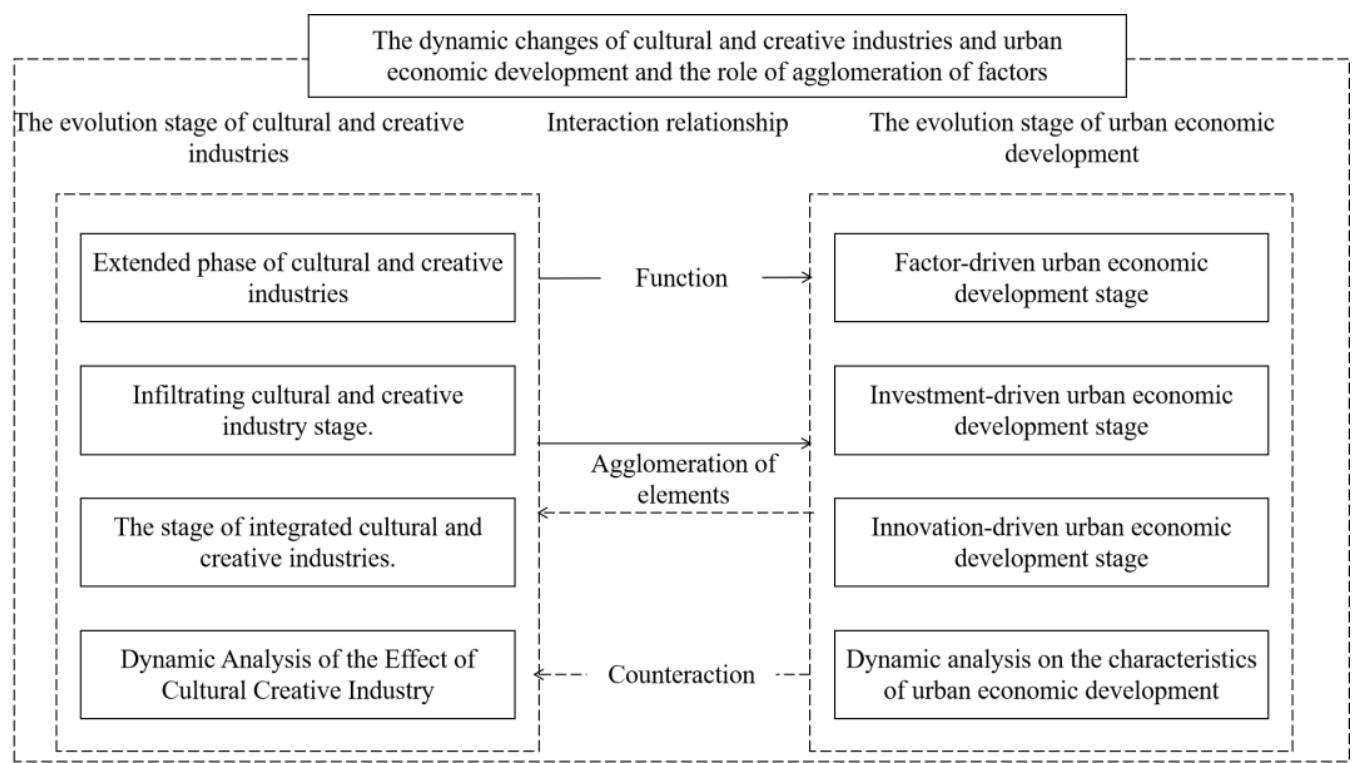

Figure 1. The interactive evolution of cultural and creative industries and urban economic development and the role of element agglomeration

The evolution and development of urban economy generally goes through three stages: factordriven urban economic development stage, investment-driven urban economic development stage and innovation-driven economic development stage, which are generally called primary stage, intermediate stage and advanced stage [8]. As a new industry that combines culture, creativity and technology, the cultural creative industry will also experience different stages of evolution during its development and evolution. Starting from the extended cultural and creative industry stage, developing to the penetrating cultural and creative industry stage, and then developing to the integrated cultural and creative industry stage, the cultural and creative industry will eventually develop into a pillar industry of the national economy.

The interaction between cultural and creative industries and urban economic development affects the existing form and operation mode of factor agglomeration. The rise and development of cultural and creative industries will attract capital, talents, land, science and technology, information and other production factors to the industry, thus forming factor aggregation effect and promoting urban economic development. At the same time, the rapid development of urban economy will form a greater siphon effect, promote the aggregation of more external production factors, and provide more required production factors for cultural and creative industries.

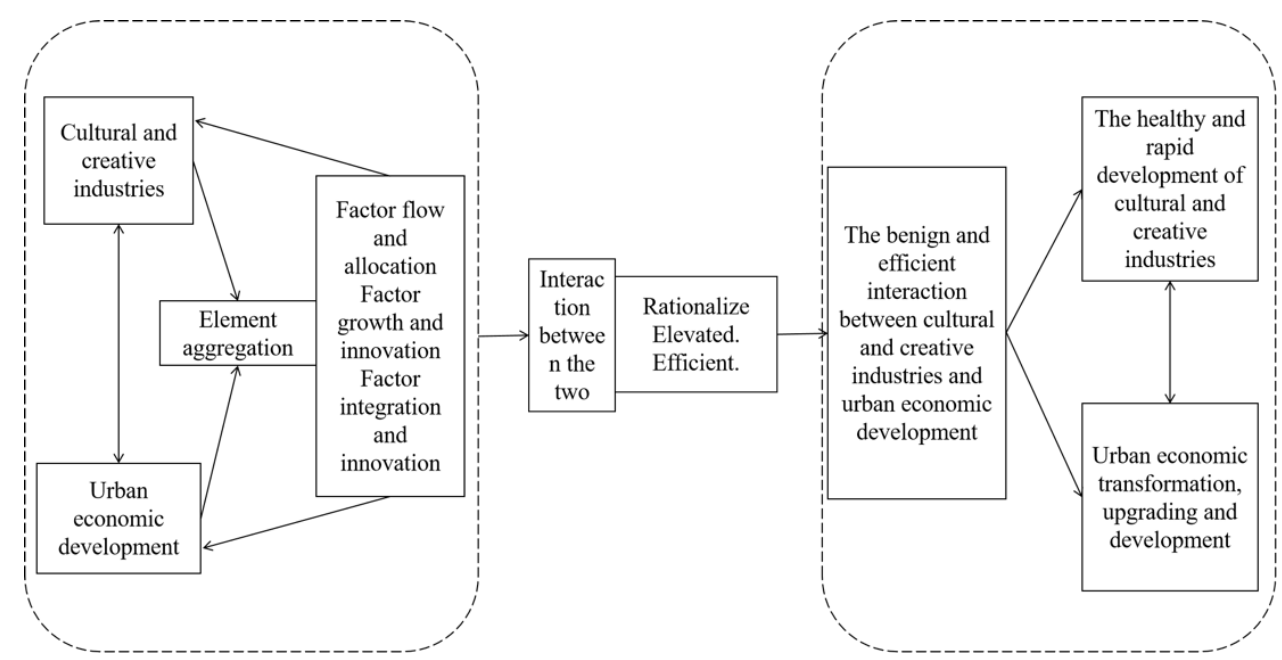

Figure 2. Mechanism and path of interaction between creative industries and urban economic development 
It can be seen from fig. 2 that the elements are influenced and interacted with each other by influencing the operation mode and existing form of aggregation. The operation mode and existing form of factor aggregation include not only the flow and allocation of factors, but also the growth innovation and integration and reorganization innovation after factor aggregation.

\section{The Role of Cultural and Creative Industries on Urban Economic Growth under the Background of Media Convergence}

The phenomenon of high market concentration widely exists in the domestic traditional cultural industry. It is worth thinking about that, on the one hand, the concentration of ownership has improved production efficiency and intensified competition, but in the long run it has led to the weakening of competition. This is also known as the "capital paradox." The cultural creative industry is centered on personal creativity. Fundamentally speaking, it is competitive, individualized, and diversified. Compared with material products, cultural products have fewer economies of scale. From the perspective of consumers, personalized consumption has also become the mainstream of future consumption. These two points are the fundamental reasons for the diversification of content supplier ownership and content product forms in the new media industry. It is the personalization and diversity of the cultural and creative industry that makes the integrated operation link a key link. The core feature of the operation platform is service, which is also the major difference between the cultural and creative industry as a service-oriented economy and an industrial economy.

At present, there are usually two modeling methods for multivariate statistical analysis of structural equations, one is the ordinary least squares method (OLS) which is commonly used in models proposed by other scholars, and the other is the partial least squares method (PLS) proposed by others. Cultural and creative industry's own industrial characteristics determine its special position in the whole national economic system, which has surpassed the single-dimensional relationship of other industries' influence on economic growth. Its emergence has made the industry's research on economic growth more complicated. The innovation mechanism, diffusion mechanism, and transformation mechanism set the internal mechanism of cultural and creative industries influencing economic growth points and the evolution path of spiritual products themselves, and they evolve through this path. The result is precisely an important factor affecting economic growth. Therefore, from this idea, we can establish the correlation model between cultural and creative industries and economic growth, and add several intermediary variables to the direct path of the impact of cultural and creative industries on economic growth, including three production factors: technological innovation, cultural capital and human capital, Thus, it constructs a new model to explain the path of cultural and creative industries affecting economic growth. As shown in Figure 3.

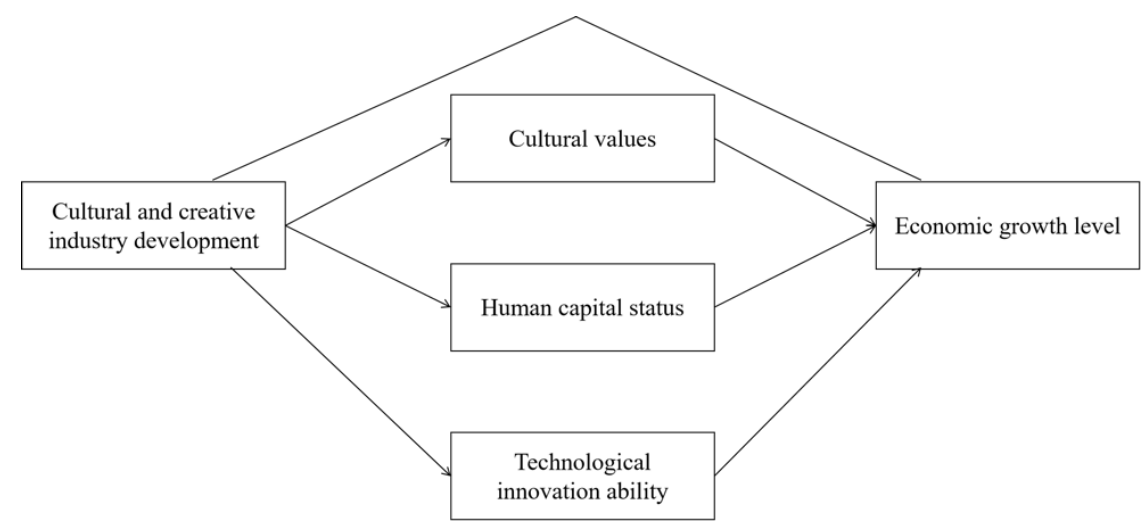

Figure 3. PLS path analysis model

When the spiritual products created by cultural and creative industries not only meet people's spiritual needs, but also become important factors affecting economic growth due to their knowledge spillover and diffusion effects, these spiritual products are no longer products in a simple sense, but 
have significant value-added effects, at which time they are transformed into a new form of capital, which we call spiritual capital. According to the state of existence of spiritual capital, spiritual capital can be divided into two categories: internal spiritual capital and external spiritual capital. The two influence and transform each other, and are closely related to the industrial structure system of the cultural and creative industries. External spiritual capital is the product of human spiritual activity or spiritual production, that is, spiritual product. It is a reflection of human beings to the objective world, a spiritual creation formed through information acquisition, processing, and processing.

There is a close and profound internal relationship and interaction between cultural and creative industries and urban economy. On the one hand, cultural and creative industries stimulate the charm and vitality of cities with their strong uniqueness and creativity, and directly constitute the source of urban economic growth. On the other hand, the competition of cultural and creative industries is becoming more prominent at the urban level. Cities with strong competitiveness and strong economic strength will provide superior conditions for the development of cultural and creative industries.

\section{Conclusion}

Today, with the vigorous development of cultural and creative industries, new media has brought stronger vitality and greater value space to traditional TV media. At the same time, the traditional TV media has brought abundant program resources to the new media, and then enhanced the profitability and social influence of the new media. Media integration, this new media pattern will surely create a more prosperous future for the cultural and creative industries. The city is an important carrier of a country's economic development. The city has the advantages of a developed economy, strong cultural consumption, and a large number of high-quality talents, and is the main gathering place for the development of cultural and creative industries. The cultural and creative industry is a relatively special emerging industry in the national economic system, which is mainly manifested in its more important and complex impact on the development of the national economy. The cultural and creative industry is the product of the development of productivity to a higher level and the consumption structure to a higher level. It is the inevitable result of the transition from the stage of material economic development to the stage of spiritual economic development. The development of cultural and creative industries plays a significant role in promoting economic growth, human capital, the improvement of technological innovation ability and the formation of cultural values. The development of cultural and creative industries in various countries and regions shows very strong growth, innovation and industrial relevance, which plays a breakthrough role in driving economic growth. In addition, cultural and creative industries have also opened up a brand-new development mode of mobilizing cultural resources and integrating economic resources, which is conducive to organically combining the economic goal of enhancing the proportion of high value-added industries with the social goal of promoting national culture.

\section{References}

[1] Zhao Wei. Research on Paper Media Content Convergence under the Background of Media Convergence --Taking "Beijing News" Economic News as an example[J]. News Research Guide, 2019, 010(008):2122, 49.

[2] Cheng Yuan. Exploration and enlightenment: the relationship between cultural and creative industries and urban economic development[J]. China Business Journal, 2016, 000(030):139-141.

[3] Ke Changbo. Research on the Interactive Mechanism of Cultural and Creative Industries and Urban Economic Development[J]. Business News, 2020, No.214(24):174-175.

[4] Xiaoling She. On the formation of the book culture industry chain under the background of Media convergence[J]. China Business Forum, 2018, 000(022):145-146.

[5] Gao Changchun, Kang Yushan. Research on the interaction between urban economic development and cultural and creative industries[J]. Times Finance, 2019(06):102-103. 
[6] Ye Juanhui. The impact of cultural and creative industries on economic growth under the background of the new normal: Taking Fuzhou as an example[J]. Journal of Fujian University of Business (formerly Journal of Fujian College of Commerce), 2016(4): 14-20.

[7] Yang Maomao, Zhu Hongxing. An Empirical Study on the Impact of Cultural and Creative Industries on Hangzhou's Economic Development[J]. Technology and Innovation Management, 2018, 039(001): 8791.

[8] Liu Chaoyou, Zhu Hongxing. Research on the Impact of Cultural and Creative Industries on Shanghai's Economic Growth [J]. Logistics Engineering and Management, 2017(06):165-167. 